\title{
Circular Stable Matching and 3-way Kidney Transplant
}

\author{
Chien-Chung Huang
}

Received: 31 December 2008 / Accepted: 13 August 2009 / Published online: 29 August 2009

(C) The Author(s) 2009. This article is published with open access at Springerlink.com

\begin{abstract}
We consider the following version of the stable matching problem. Suppose that men have preferences for women, women have preferences for dogs, and dogs have preferences for men. The goal is to organize them into family units so that no three of them have incentive to desert their assigned family members to join in a new family. This problem is called circular stable matching, allegedly originated by Knuth. We also investigate a generalized version of this problem, in which every participant has preference among all others. The goal is similarly to partition them into oriented triples so that no three persons have incentive to deviate from the assignment. This problem is motivated by recent innovations in kidney exchange, and we call it the 3-way kidney transplant problem. We report complexity, structural and counting results on these two problems.
\end{abstract}

\section{Introduction}

Stable matching problems were introduced by Gale and Shapley in their seminal paper [6]. Knuth asked whether the stable matching problem can be extended to the case of three parites [15], say we have women, men, and dogs. This fairly general problem allows several formulations. One possibility is that every player expresses her/his/its preference among the combinations of the other two parties. In this formulation, $\mathrm{Ng}$ and Hirschberg [16] proved the existence of stable matchings is NP-complete. Similar NP-completeness results have been shown in $[10,21]$.

$\mathrm{Ng}$ and Hirschberg mentioned that the reviewers of their paper suggest another formulation, and they attributed it to Knuth, for the 3-party stable matchings- the CIRCULAR STABLE MATCHING problem that we will consider in this paper-women

C.-C. Huang $(\bowtie)$

Max-Planck-Institute für Informatik, Saarbrücken, Germany

e-mail: villars@mpi-inf.mpg.de 


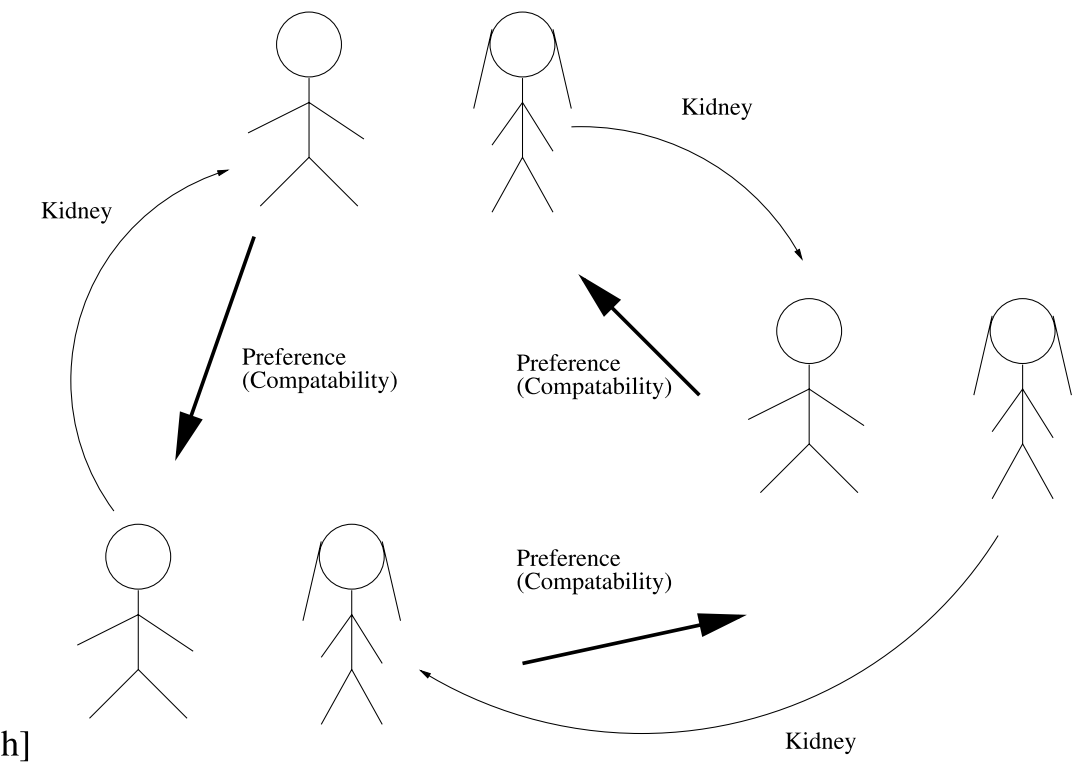

Fig. 1 An illustration of kidney exchange with compatibility as preference

have preferences for dogs, dogs have preferences for men, and men have preferences for women. The goal is to organize them into stable family units so that people/dogs have no incentive to desert their assigned family members to join in a new family. This problem can be seen as a natural generalization of the well-known 2-party STABLE MARRIAGE problem and have been investigated in $[3,5]$.

A generalized version of the CIRCULAR STABLE MATCHING problem allows each participant to express preference among all others. The goal is to partition $3 n$ persons into oriented triples so that no three of them have reasons to deviate from the assignment. Again, this problem can be regarded as a generalization of the STABLE ROOMMATES problem [6]. This generalized problem has practical interest in the kidney exchange that has received much attention recently $[1,4,8,12,17-20]$. The "preference" here can be interpreted as degrees of compatibility between recipients and donors. Figure 1 gives a more visual way of seeing the connection between circular matching and kidney exchange. In this paper, we call this problem the 3-WAY KIDNEY TRANSPLANT problem. For ease of presentation, we will refer to all participants in both problems generically as "players."

The two problems require a proper definition of stability. In the two-party STABLE MARRIAGE and STABLE ROOMMATES, a matching is stable if there is no blocking pair: two persons who strictly prefer each other to their assigned partners. Naturally, one would extend blocking pairs into blocking triples to define the stability of matchings. However, a blocking triple here is more tricky. To see why this is so, consider the following. 
- In CIRCULAR STABLE MATCHING, suppose that we have a matching $\left\{\left(m_{1}, w_{1}, d_{1}\right)\right.$, $\left.\left(m_{2}, w_{2}, d_{2}\right),\left(m_{3}, w_{3}, d_{3}\right)\right\}$. If $m_{1}$ prefers $w_{2}$ to $w_{1}, w_{2}$ prefers $d_{3}$ to $d_{2}$, and $d_{3}$ prefers $m_{1}$ to $m_{3}$, then $\left(m_{1}, w_{2}, d_{3}\right)$ is clearly a blocking triple. But it may also be the case that $w_{2}$ prefers $d_{1}$ to $d_{2}$. Then $\left(m_{1}, w_{2}, d_{1}\right)$ can also be regarded as a (weaker) blocking triple, since only $m_{1}$ and $w_{2}$ are really better off in such a triple, while $d_{1}$ is indifferent.

- In 3-WAY KIDNEY TRANSPLANT, a matching is composed of oriented triples. Here we write such a triple as $\left(k_{1}, k_{2}, k_{3}\right)$ to express that $k_{2}, k_{3}, k_{1}$ are the successors of $k_{1}, k_{2}, k_{3}$, respectively. Moreover, here $k_{1}$ represents a couple (often a married couple) consisting of a person needing a new kidney and a potential kidney donor. If $k_{2}$ follows $k_{1}$ in a triple, then the donor from the couple $k_{2}$ will be passing a kidney to the recipient of $k_{1}$. Thus, it is $k_{1}$ 's preference (degree of compatibility) that is at issue. Note that an oriented couple $\left(k_{1}, k_{2}, k_{3}\right)$ can be a blocking triple itself $\left(k_{1}, k_{3}, k_{2}\right)$, if $k_{1}$ prefers $k_{3}$ to $k_{2}, k_{3}$ prefers $k_{2}$ to $k_{1}$, and $k_{2}$ prefers $k_{1}$ to $k_{3}$. Such phenomena may appear somehow surprising for researchers long familiar with stable matching literature.

We allow players to express their indifferences in the form of ties in the preference lists. Now we say a blocking triple is of degree $i$ if $i$ players are strictly better off in such a triple than in a given matching, while the remaining $3-i$ players are indifferent. Note that the indifference can be either because the involved player is still matched to the same partner (or still having the same successor in the oriented triple), or because the involved player has a partner/successor who is tied with her/his/its current assignment. We define a hierarchy of stabilities (which is similar to the one defined by Irving [11] in the 2-party matching) as follows.

- Super Stable Matching: a matching not allowing blocking triples of degree 1 or 2 or 3 .

- Strong Stable Matching: a matching not allowing blocking triples of degree 2 nor those of degree 3 .

- Weak Stable Matching: a matching not allowing blocking triples of degree 3.

Contributions of the Paper

Complexity: We prove the following existence problems are NP-complete: super/strong stable matchings in CIRCULAR STABLE MATCHING; super/strong/weak stable matchings in 3-WAY KIDNEY TRANSPLANT. Therefore, it is unlikely that we can design efficient algorithms to solve these problems. The complexity of weak stable matchings in CIRCULAR STABLE MATCHING remains open. However, there is empirical evidence indicating that it probably does not belong to the class of NPcomplete problems. We shall discuss this issue later.

Independently, Biró and McDermid [2] obtained similar NP-completeness results for both of problems studied in this work.

Structural Results: It is well-known that stable matchings in 2-party stable marriage and stable roommates have rich structures and sophisticated algorithms have been designed to exploit them $[9,15]$. It turns out that strong stable matchings in CIRCULAR 
STABLE MATCHING have parallel (but even richer) structures. Briefly, we show that the set of strong stable matchings form a union of distributive lattices.

Counting Results: We prove that counting strong stable matchings in both problems is \#P-complete. Moreover, the number of strong and weak stable matchings in both problems can be exponential.

\section{Notation and Paper Roadmap}

In the paper, we use $\mathcal{M}, \mathcal{W}, \mathcal{D}$ to denote the collections of men and women and dogs in CIRCULAR STABLE MATCHING. Whatever the problem instance, we will always assume that they are of the same cardinality. Similarly, $\mathcal{K}$ means the set of players in 3-way kidney transplant. $P(p)$ denotes the preference list of player $p$. The notation $\succ$ indicates the preference order in the list. The braces denote a tie. For example, $P(m)=\left\{w_{1}, w_{2}\right\} \succ w_{3}$ means that man $m$ prefers both $w_{1}$ and $w_{2}$ to $w_{3}$ while he is indifferent between the former two. In general, we use $\mu$ to denote a 3-dimensional matching (consisted of triples). We will need to consider the induced two-party matching of $\mu$. For example, we write $\left.\mu\right|_{\mathcal{M}, \mathcal{W}}$ to denote the induced menwomen matching by dropping all dogs from the triples of $\mu$. Finally, $\pi_{r}(X)$ denotes an arbitrary permutation of the members in the set $X$.

Section 2 presents complexity results; Sect. 3 reports structural results of stable matching; Sect. 4 concerns the counting of stable matchings. Finally, Sect. 5 draws conclusions.

\section{NP-completeness of Strong Stable Matchings}

The reductions we will present share similar ideas to those used in [10]. The main difference lies in the design of "guard players" (to be explained below).

\subsection{Existence Problem of Super Stable Matchings is NP-complete}

To prove that the existence of super stable matchings is NP-complete in circular stable matching, we present a reduction from 3-DIMENSIONAL MATCHING, one of Karp's 21 NP-complete problems [14]. The problem instance is given in the form $\Upsilon=(\mathcal{M}, \mathcal{W}, \mathcal{D}, \mathcal{T})$, where $\mathcal{T} \subseteq \mathcal{M} \times \mathcal{W} \times \mathcal{D}$. The goal is to decide whether a perfect matching $\mu \subseteq \mathcal{T}$ exists. This problem remains NP-complete even if every player in $\mathcal{M} \cup \mathcal{W} \cup \mathcal{D}$ appears exactly 2 or 3 times in the triples of $\mathcal{T}$ [7].

We first explain the intuition behind our reduction. Supposing that man $m_{i}$ appears in three triples $\left(m_{i}, w_{i a}, d_{i a}\right),\left(m_{i}, w_{i b}, d_{i b}\right),\left(m_{i}, w_{i c}, d_{i c}\right)$ in $\mathcal{T}$, we create three dopplegangers, $m_{i 1}, m_{i 2}, m_{i 3}$ in the derived circular stable matching instance with ties $\Upsilon^{\prime}$. We also create four garbage collectors, $w_{i 1}^{g}, d_{i 1}^{g}, w_{i 2}^{g}, d_{i 2}^{g}$. The aim of our design is that in the derived instance $\Upsilon^{\prime}$, in a super stable matching, exactly one doppleganger will be matched to a woman-dog pair with whom $m_{i}$ shares a triple in $\mathcal{T}$, while the other two dopplegangers will be paired off with garbage collectors. In the case that there are only two triples in $\mathcal{T}$ containing man $m_{j}$, we create only 
Table 1 The preference lists of all players in the derived instance $\Upsilon^{\prime}$. Recall that \{\} denotes a tie in the preferences. Note also that real women $\mathcal{W}$ and real dogs $\mathcal{D}$ only list real dogs and dopplegangers, respectively, with whom they share triples in $\mathcal{T}$, at the top of their lists

\begin{tabular}{|c|c|}
\hline Major players & Preference lists \\
\hline$m_{i 1} \in \mathcal{M}_{1}$ & $\left\{w_{i 1}^{g}, w_{i 2}^{g}, w_{i a}\right\} \succ w^{\star}\left(m_{i 1}\right) \succ \cdots$ \\
\hline$m_{i 2} \in \mathcal{M}_{2}$ & $\left\{w_{i 1}^{g}, w_{i 2}^{g}, w_{i b}\right\} \succ w^{\star}\left(m_{i 2}\right) \succ \cdots$ \\
\hline$m_{i 3} \in \mathcal{M}_{3}$ & $\left\{w_{i 1}^{g}, w_{i 2}^{g}, w_{i c}\right\} \succ w^{\star}\left(m_{i 3}\right) \succ \cdots$ \\
\hline$w \in \mathcal{W}$ & $\{d \mid(*, w, d) \in \mathcal{T}\} \succ d^{\star}(w) \succ \cdots$ \\
\hline$d \in \mathcal{D}$ & $\left\{m_{i j} \mid\left(m_{i}, w, d\right) \in \mathcal{T}, w \succ_{m_{i j}} w^{\star}\left(m_{i j}\right)\right\} \succ m^{\star}(d) \succ \cdots$ \\
\hline$w_{i 1}^{g} \in \mathcal{W}_{1}^{g}$ & $d_{i 1}^{g} \succ d^{\star}\left(w_{i 1}^{g}\right) \succ \cdots$ \\
\hline$w_{i 2}^{g} \in \mathcal{W}_{2}^{g}$ & $d_{i 2}^{g} \succ d^{\star}\left(w_{i 2}^{g}\right) \succ \cdots$ \\
\hline$d_{i 1}^{g} \in \mathcal{D}_{1}^{g}$ & $\left\{m_{i 1}, m_{i 2}, m_{i 3}\right\} \succ m^{\star}\left(d_{i 1}^{g}\right) \succ \cdots$ \\
\hline$d_{i 2}^{g} \in \mathcal{D}_{2}^{g}$ & $\left\{m_{i 1}, m_{i 2}, m_{i 3}\right\} \succ m^{\star}\left(d_{i 2}^{g}\right) \succ \cdots$ \\
\hline Guard players & Preference lists \\
\hline$m^{\star}\left(m^{\dagger}\right), m^{\dagger} \in \mathcal{M}_{1} \cup \mathcal{M}_{2} \cup \mathcal{M}_{3}$ & $w^{\star}\left(m^{\dagger}\right) \succ \cdots$ \\
\hline$w^{\star}\left(m^{\dagger}\right), m^{\dagger} \in \mathcal{M}_{1} \cup \mathcal{M}_{2} \cup \mathcal{M}_{3}$ & $d^{\star}\left(m^{\dagger}\right) \succ \cdots$ \\
\hline$d^{\star}\left(m^{\dagger}\right), m^{\dagger} \in \mathcal{M}_{1} \cup \mathcal{M}_{2} \cup \mathcal{M}_{3}$ & $\left\{m^{\dagger}, m^{\star}\left(m^{\dagger}\right)\right\} \succ \cdots$ \\
\hline$m^{\star}\left(w^{\dagger}\right), w^{\dagger} \in \mathcal{W}_{1}^{g} \cup \mathcal{W}_{2}^{g} \cup \mathcal{W}$ & $\left\{w^{\dagger}, w^{\star}\left(w^{\dagger}\right)\right\} \succ \cdots$ \\
\hline$w^{\star}\left(w^{\dagger}\right), w^{\dagger} \in \mathcal{W}_{1}^{g} \cup \mathcal{W}_{2}^{g} \cup \mathcal{W}$ & $d^{\star}\left(w^{\dagger}\right) \succ \cdots$ \\
\hline$d^{\star}\left(w^{\dagger}\right), w^{\dagger} \in \mathcal{W}_{1}^{g} \cup \mathcal{W}_{2}^{g} \cup \mathcal{W}$ & $m^{\star}\left(w^{\dagger}\right) \succ \cdots$ \\
\hline$m^{\star}\left(d^{\dagger}\right), d^{\dagger} \in \mathcal{D}_{1}^{g} \cup \mathcal{D}_{2}^{g} \cup \mathcal{D}$ & $w^{\star}\left(d^{\dagger}\right) \succ \cdots$ \\
\hline$w^{\star}\left(d^{\dagger}\right), d^{\dagger} \in \mathcal{D}_{1}^{g} \cup \mathcal{D}_{2}^{g} \cup \mathcal{D}$ & $\left\{d^{\star}\left(d^{\dagger}\right), d^{\dagger}\right\} \succ \cdots$ \\
\hline$d^{\star}\left(d^{\dagger}\right), d^{\dagger} \in \mathcal{D}_{1}^{g} \cup \mathcal{D}_{2}^{g} \cup \mathcal{D}$ & $m^{\star}\left(d^{\dagger}\right) \succ \cdots$ \\
\hline
\end{tabular}

2 dopplegangers $m_{j 1}, m_{j 2}$ and two garbage collectors $w_{j 1}^{g}, d_{j 1}^{g}$. Similarly, the intent is to make sure that in a super stable matching, exactly one doppleganger will be matched to a woman-dog pair with whom $m_{j}$ shares a triple in $\mathcal{T}$ while the other is matched to the garbage collectors.

Now, we will refer to the set of dopplegangers as $\mathcal{M}_{1}, \mathcal{M}_{2}, \mathcal{M}_{3}$, the set of garbage collectors as $\mathcal{W}_{1}^{g}, \mathcal{W}_{2}^{g}, \mathcal{D}_{1}^{g}, \mathcal{D}_{2}^{g}$ and the original set of real women and real dogs as $\mathcal{W}, \mathcal{D}$. Collectively, we refer to them as major players $\Sigma=\mathcal{M}_{1} \cup \mathcal{M}_{2} \cup \mathcal{M}_{3} \cup \mathcal{W}_{1}^{g} \cup$ $\mathcal{W}_{2}^{g} \cup \mathcal{W} \cup \mathcal{D}_{1}^{g} \cup \mathcal{D}_{2}^{g} \cup \mathcal{D}$ and their preferences are summarized in the left column of Table 1.

To restrict the possible partners of major players in $\Sigma$, we introduce a set of gadgets called guard players. They are denoted as $m^{\star}(p), w^{\star}(p), d^{\star}(p)$, for $p \in \Sigma$ and their preferences are shown in the right column of Table 1 . Their purpose is to ensure that player $p$, say $p=m_{i 1}$, will never get a partner ranking lower than his associated guard player $w^{\star}\left(m_{i 1}\right)$ in a super stable matching. How guard players and major players interact is captured by the following lemma.

Lemma 1 In the derived instance $\Upsilon^{\prime}$, if a super stable matching exists, then in such a matching, (1) all major players in $\Sigma$ will be matched to other major players ranking higher than her/his/its associated guard players, (2) the set of guard players 
$m^{\star}(p), w^{\star}(p), d^{\star}(p)$, where $p \in \Sigma$ are matched to one another, and (3) the garbage collectors created for a particular man $m_{i}$ will be matched to one another and the two dopplegangers of $m_{i}$ (or just one if $m_{i}$ only appears twice in the triples of the given 3-dimensional matching instance $\Upsilon$ ).

Proof Without loss of generality, consider the major player $p=m_{i 1}$. In a super stable matching, if $m_{i 1}$ is matched to a woman ranking below $w^{\star}\left(m_{i 1}\right)$, then $\left(m_{i 1}, w^{\star}\left(m_{i 1}\right), d^{\star}\left(m_{i 1}\right)\right)$ is a blocking triple of degree at least 1 , a contradiction. If $m_{i 1}$ is matched to $w^{\star}\left(m_{i 1}\right)$, then $\left(m^{\star}\left(m_{i 1}\right), w^{\star}\left(m_{i 1}\right), d^{\star}\left(m_{i 1}\right)\right)$ is a blocking triple of degree at least 1 , again a contradiction.

For the second part, by the above discussion, we know that all major players must be matched to one another. Hence, if $\left(m^{\star}(p), w^{\star}(p), d^{\star}(p)\right)$ is not part of a super stable matching, they form a blocking triple of degree at least 1 .

The third part follows straightforwardly from the previous two.

Lemma 2 The given instance $\Upsilon=(\mathcal{M}, \mathcal{W}, \mathcal{D}, \mathcal{T})$ contains a perfect matching if and only if the derived instance $\Upsilon^{\prime}$ allows a super stable matching.

Proof (Sufficiency) If the derived instance $\Upsilon^{\prime}$ allows a super stable matching, then by the third part of Lemma 1 , it is easy to see that $\Upsilon$ contains a perfect matching.

(Necessity) Suppose that $\mu$ is a perfect matching in $\Upsilon$. We construct a super stable matching $\mu^{\prime}$ for the derived instance $\Upsilon^{\prime}$ as follows. Assuming that $\left(m_{i}, w_{x}, d_{y}\right) \in \mu$, we choose the doppleganger $m_{i j}$ who ranks $w_{x}$ higher than his guard player $w^{\star}\left(m_{i j}\right)$ and make $\left(m_{i j}, w_{x}, d_{y}\right)$ a triple in $\mu^{\prime}$. Further, the other two dopplegangers of $m_{i}$ are matched to $\left(w_{i 1}^{g}, d_{i 1}^{g}\right)$ and $\left(w_{i 2}^{g}, d_{i 2}^{g}\right)$ respectively. (If there are only two dopplegangers of $m_{i}$, then the other doppleganger $m_{i j^{\prime}} \neq m_{i j}$ is matched to $w_{i 1}^{g}, d_{i 1}^{g}$.) Finally, let the three guard players created for a particular major player be matched to one another. By this construction, it can be verified that we only allow blocking triples of degree 0 , which are permissible for a super stable matching.

Theorem 1 Deciding whether a super stable matching exists in a circular stable matching problem with ties in the preferences is NP-complete. This is true even if all ties are of size at most 3 and they are at the front of the preference lists.

To prove the existence of strong stable matching is NP-complete, we can use the same reduction as above with just one alteration: we need a different set of guard players for each major player. Note that in the proof of Lemma 1, we rely on blocking triples of degree 1; those are not counted as blocking triples based on the definition of strong stable matching.

The design of guard players for the reduction of strong stable matching is similar to those used in a reduction in Sect. 2.3, so we omit the details here. ${ }^{1}$

\footnotetext{
${ }^{1}$ However, in our reduction, ties are allowed. Biró and McDermid gave a stronger reduction showing that the existence of strong stable matchings is NP-complete even with strictly-ordered preference lists.
} 


\subsection{Strong/Super Stability in 3-way Kidney Transplant}

We now present a reduction from a circular stable matching instance $\Upsilon=(\mathcal{M}, \mathcal{W}$, $\mathcal{D}, \mathcal{L}$ ) (with or without ties in the preferences) to a 3-way kidney transplant instance $\Upsilon^{\prime}$. Suppose that $m \in \mathcal{M}, w \in \mathcal{W}, d \in \mathcal{D}$ have preferences $P(m), P(w), P(d)$, respectively. In $\Upsilon^{\prime}$, their preferences are transformed into

- $P^{\prime}(m)=P(m) \succ \pi_{r}(\mathcal{D}) \succ \pi_{r}(\mathcal{M}-\{m\})$.

- $P^{\prime}(w)=P(w) \succ \pi_{r}(\mathcal{M}) \succ \pi_{r}(\mathcal{W}-\{w\})$.

- $P^{\prime}(d)=P(d) \succ \pi_{r}(\mathcal{W}) \succ \pi_{r}(\mathcal{D}-\{d\})$.

To prove this is a valid reduction, we have to argue that strong/super stable matchings exist in $\Upsilon$ if and only if they exist in $\Upsilon^{\prime}$. It is straightforward to show one direction (from $\Upsilon$ to $\Upsilon^{\prime}$ ), but the other direction takes some argument.

Lemma 3 If a strong/super stable matching $\mu^{\prime}$ exists in $\Upsilon^{\prime}$, the following holds

- Every oriented triple contains exactly one man, one woman, and one dog.

- Given a triple $t \in \mu^{\prime}, t$ 's orientation must be $t=(m, w, d)$.

Proof For the first part, without loss of generality, assume that a triple $t \in \mu^{\prime}$ contains at least two men. There are three possible cases and all lead to contradiction.

1. Suppose that $t=\left(m, m^{\prime}, m^{\prime \prime}\right)$. Then there exist two triples $t^{\prime}$ and $t^{\prime \prime}$, which contain two women and two dogs, respectively. As a result, a woman $w \in t^{\prime}$ and a $\operatorname{dog}$ $d \in t^{\prime \prime}$ have as successors a woman, and a dog, respectively. Similarly, there is a man $m \in t$ whose successor is another man. Then $(m, w, d)$ is a blocking triple of degree 3 , violating the stability of $\mu^{\prime}$.

2. Suppose that $t=\left(m, m^{\prime}, w\right)$. Then there exists a triple $t^{\prime}$ containing two dogs. At least one $\operatorname{dog} d \in t^{\prime}$ has another $\operatorname{dog}$ as successor. Then $(m, w, d)$ is a blocking triple of degree 3, blocking $\mu^{\prime}$.

3. Suppose that $t=\left(m, m^{\prime}, d\right)$. Then the argument is analogous to the previous case.

For the second part, if $t=(m, d, w) \in \mu^{\prime}$, then the reverse triple $(m, w, d)$ is a blocking triple of degree 3 .

By Lemma 3, the following theorem is immediate.

Theorem 2 It is NP-complete to decide whether a strong/super stable matching exists in the 3-way kidney transplant problem.

\subsection{Weak Stability in 3-way Kidney Transplant}

The reduction we are presenting in this section shares similar basic ideas to those we used in Sect. 2.1: reduction from a 3-dimensional matching problem instance $\Upsilon=(\mathcal{M}, \mathcal{W}, \mathcal{D}, \mathcal{L})$, creating dopplegangers $\mathcal{M}_{1} \cup \mathcal{M}_{2} \cup \mathcal{M}_{3}$ and garbage collectors $\mathcal{W}_{1}^{g} \cup \mathcal{W}_{2}^{g} \cup \mathcal{D}_{1}^{g} \cup \mathcal{D}_{2}^{g}$, and using sets of guard players to restrict the potential partners (successors in triples) of the major players. The key difference is the design of the guard players' preferences. 
We introduce the following gadget for each major player $k \in \mathcal{M}_{1} \cup \mathcal{M}_{2} \cup \mathcal{M}_{3} \cup$ $\mathcal{W}_{1}^{g} \cup \mathcal{W}_{2}^{g} \cup \mathcal{D}_{1}^{g} \cup \mathcal{D}_{2}^{g}$. (Note that real women $\mathcal{W}$ and real dogs $\mathcal{D}$ do not need them.) Let $\Upsilon_{k}$ be a 3-way kidney transplant instance that has the following three properties: (1) It contains 7 players, $k_{i}^{\#}, 1 \leq i \leq 7$, (2) it does not allow any weak stable matching, and (3) if one player, $k_{1}^{\#}$, is removed from $\Upsilon_{k}$, then the remaining 6 players' preferences allow at least one weak stable matching. Such an instance $\Upsilon_{k}$ can be found in Appendix A. Our plan is to "embed" instances $\Upsilon_{k}$ into the intended 3-way kidney transplant instance $\Upsilon^{\prime}$.

We now explain in more detail what we mean by embedding of $\Upsilon_{k}$ into $\Upsilon^{\prime}$. For illustration, we first show the preferences of $m_{i 1}$ and his six associated guard players in $\Upsilon^{\prime}$.

- $P_{\Upsilon^{\prime}}\left(m_{i 1}\right)=w_{i 2}^{g} \succ w_{i 1}^{g} \succ w_{i a} \succ P_{\Upsilon_{m_{i 1}}}\left(m_{i 1,1}^{\#}\right) \succ \cdots$, where $P_{\Upsilon_{m_{i 1}}}\left(m_{i 1,1}^{\#}\right)$ is the preference list of $m_{i 1,1}^{\#}$ in the instance $\Upsilon_{m_{i 1}}$.

- $P_{\Upsilon^{\prime}}\left(m_{i 1, j}^{\#}\right)=P_{\Upsilon_{m_{i 1}}}\left(m_{i 1, j}^{\#}\right) \succ \cdots$, where $2 \leq j \leq 7$ and $P_{\Upsilon_{m_{i 1}}}\left(m_{i 1, j}^{\#}\right)$ is the preference list of $m_{i 1, j}^{\#}$ in the instance $\Upsilon_{m_{i 1}}$.

In words, as $k=m_{i 1}$, we let $m_{i 1}$ "play the role" of $k_{1}^{\#}\left(=m_{i 1,1}^{\#}\right)$. His associated six guard players in $\Upsilon_{k}\left(=\Upsilon_{m_{i 1}}\right)$ are added into $\Upsilon^{\prime}$ and, in their new preferences, they still put one another on top of their lists. By this arrangement, if $m_{i 1}$ can be matched to some woman ranking higher than his associated guard players, then in this sense, $m_{i 1,1}^{\#}\left(=m_{i 1}\right)$ is removed from the problem instance $\Upsilon_{m_{i 1}}$; on the other hand, if he is not, then $\Upsilon_{m_{i 1}}$ will engender at least a blocking triple, disrupting the stability of the matching in $\Upsilon^{\prime}$.

Lemma 4 In a weak stable matching $\mu^{\prime}$ in $\Upsilon^{\prime}$, the successor of $m_{i 1}$ ranks at least as high as $w_{i a}$. Moreover, the six guard players of $m_{i 1}$ must be matched to one another.

Proof If $m_{i 1}$ is matched to someone ranking lower than $w_{i a}$, then whatever the oriented triples of $\mu^{\prime}$ involving the six guard partners of $m_{i 1}$ and $m_{i 1}$ himself, the situation is identical to one where we have a matching $\mu^{\phi}$ for the problem instance $\Upsilon_{m_{i 1}}$, which by design, involves at least one blocking triple of degree 3 to block $\mu^{\phi}$, and also $\mu^{\prime}$. The second part of the lemma follows from the first part and the way we chose the gadget $\Upsilon_{k}\left(=\Upsilon_{m_{i 1}}\right)$.

The detailed preferences of major players can be found in Table 2. Note that Lemma 4 also applies to other major players who have associated guard players. Thus, in a weak stable matching, they will get a successor ranking strictly higher than their guard players.

Theorem 3 Deciding whether a weak stable matching exists in a 3-way kidney transplant problem is NP-complete.

Proof By Lemma 4, if $\mu^{\prime}$ is a weak stable matching in $\Upsilon^{\prime}$, we can throw away triples involving guard players of $\Upsilon^{\prime}$, along with the garbage collectors (and the dopplegangers matched to them). Replace the doppleganger $m_{i j}$ with the real man $m_{i}$ gives the desired perfect matching $\mu$ in $\Upsilon$. 
Table 2 The preference lists of major players in the derived problem instance $\Upsilon^{\prime}$

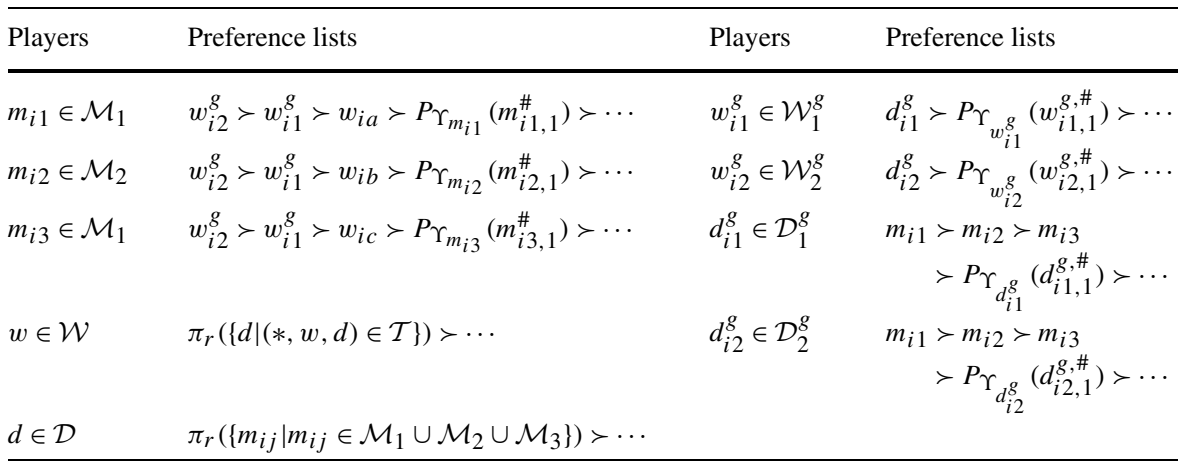

For the other direction, we will construct a weak stable matching $\mu^{\prime}$ in $\Upsilon^{\prime}$ based on a perfect matching $\mu$ in $\Upsilon$. Suppose that $\left(m_{i}, w_{x}, d_{y}\right) \in \mu$. In $\mu^{\prime}$, we insert three triples, $\left(m_{i j}, w_{x}, d_{y}\right)$, where $m_{i j}$ is the doppleganger of $m_{i}$ who ranks $w_{x}$ higher than his guard players, and $\left(m_{i j^{\prime}}, w_{i 1}^{g}, d_{i 1}^{g}\right)$ and $\left(m_{i j^{\prime \prime}}, w_{i 2}^{g}, d_{i 2}^{g}\right)$. (Or we only add the first two triples, provided that $m_{i}$ only appears twice in the triples of $\mathcal{T}$.) It can be observed that $\mu^{\prime}$ involves only blocking triples of degree at most 2 , which are allowed because of the definition of weak stable matchings.

\section{Structures of Strong Stable Matchings}

We first review the definition of distributive lattices.

Definition 1 Let $(\mathcal{E}, \preceq)$ be a poset. Such a poset is a distributive lattice if it fulfills the following three properties:

1. Each pair of elements $a, b \in \mathcal{E}$ has an infimum, called meet, denoted as $a \wedge b \in \mathcal{E}$, such that $a \wedge b \preceq a, a \wedge b \preceq b$, and there is no element $c \in \mathcal{E}$ such that $c \preceq a, c \preceq b$, and $a \wedge b \succ c$.

2. Each pair of elements $a, b \in \mathcal{E}$ has a supremum, called join, denoted as $a \vee b \in \mathcal{E}$, such that $a \preceq a \vee b, b \preceq a \vee b$, and there is no element $c \in \mathcal{E}$ such that $a \preceq c, b \preceq c$, and $c \succ a \vee b$.

3. Given any three elements, $a, b, c \in \mathcal{E}$, the distributive law holds, i.e., $a \wedge(b \vee c)=$ $(a \wedge b) \vee(a \wedge c)$, and $a \vee(b \wedge c)=(a \vee b) \wedge(a \vee c)$.

Note that in this section, we assume that all preference lists are strictly ordered.

Our major finding regarding the structure of strong stable matchings in CIRCULAR STABLE MATCHING is that they are a collection of distributive lattices. In particular, consider the subset of strong stable matchings in which all players in one group (men, women, or dogs) have the same partners. Such a subset is a distributive lattice. The following theorem gives a more precise statement.

Theorem 4 Let $\Upsilon=(\mathcal{M}, \mathcal{W}, \mathcal{D}, \mathcal{P})$ be a circular stable matching instance and let the set of strong stable matchings in $\Upsilon$ be denoted as $\Omega$. Given a two-party matching 
$N_{\mathcal{P}, \mathcal{Q}}=\left\{\left(p_{i 1}, q_{i 1}\right),\left(p_{i 2}, q_{i 2}\right), \ldots,\left(p_{i n}, q_{i n}\right)\right\}$ where $p_{i j} \neq p_{i j^{\prime}}, q_{i j} \neq q_{i j^{\prime}}, p_{i j} \in \mathcal{P}$, $q_{i j} \in \mathcal{Q}, \mathcal{P}, \mathcal{Q} \in\{\mathcal{M}, \mathcal{W}, \mathcal{D}\}, \mathcal{P} \neq \mathcal{Q}$, the subset of strong stable matchings $\Omega_{N_{\mathcal{P}, \mathcal{Q}}}=$ $\left\{\mu|\mu \in \Omega, \mu|_{\mathcal{P}, \mathcal{Q}}=N_{\mathcal{P}, \mathcal{Q}}\right\}$ is a distributive lattice.

We make two remarks here. First, when we consider a non-empty subset $\Omega_{N_{\mathcal{P}, \mathcal{Q}}}=$ $\Omega_{N_{\mathcal{M}, \mathcal{W}}}$ of strong stable matchings, we impose a partial order on the elements based on the welfare of one particular group, which, in this case, is $\mathcal{W}$. (Note that all men

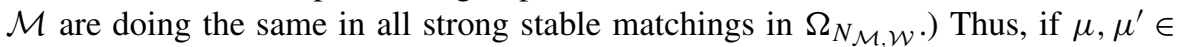
$\Omega_{N_{\mathcal{M}, \mathcal{W}}}$, then $\mu \succ \mu^{\prime}$ if and only if all women in $\mathcal{W}$ are getting dogs in $\mu$ ranking at least as high as those they get in $\mu^{\prime}$. Second, if $\Omega_{N_{\mathcal{P}, \mathcal{Q}}}=\emptyset$, we are assuming that it is (vacuously) a distributive lattice.

Lemma 5 Let $\mu$ and $\mu^{\prime}$ be two strong stable matchings in $\Omega_{N_{\mathcal{D}, \mathcal{M}}}$ and man $m$ and woman $w$ belong to the same triple in $\mu$ but not so in $\mu^{\prime}$. Then one of them prefers $\mu$ while the other prefers $\mu^{\prime}$.

Proof Let $\mathcal{X}, \mathcal{Y}$ be the sets of men and women preferring $\mu$ respectively; analogously, let $\mathcal{X}^{\prime}, \mathcal{Y}^{\prime}$ be the set of men and women preferring $\mu^{\prime}$ respectively.

We claim that if $m \in \mathcal{X}$, then his partner $w$ in $\mu$ must be a member of $\mathcal{Y}^{\prime}$. If this is not so, then $(m, w, d)$ blocks $\mu^{\prime}$, where $d$ is the dog that has $m$ as a partner in both $\mu$ and $\mu^{\prime}$. Thus, we have $|\mathcal{X}| \leq\left|\mathcal{Y}^{\prime}\right|$. By an analogous argument, every man $m$ in $\mathcal{X}^{\prime}$ must have a woman $w \in \mathcal{Y}$ as a partner in $\mu^{\prime}$, otherwise, $(m, w, d)$ blocks $\mu$, where $d$ is the dog that has $m$ as a partner in both $\mu$ and $\mu^{\prime}$. So we have $\left|\mathcal{X}^{\prime}\right| \leq|\mathcal{Y}|$.

By the fact that in both $\mu$ and $\mu^{\prime}$, all dogs have the same partners, so the number of men and women having different partners must be equal: $|\mathcal{X}|+\left|\mathcal{X}^{\prime}\right|=|\mathcal{Y}|+\left|\mathcal{Y}^{\prime}\right|$. This, combined with the previous two facts, $|\mathcal{X}| \leq\left|\mathcal{Y}^{\prime}\right|$ and $\left|\mathcal{X}^{\prime}\right| \leq|\mathcal{Y}|$, implies that $|\mathcal{X}|=\left|\mathcal{Y}^{\prime}\right|,|\mathcal{X}|=\left|\mathcal{Y}^{\prime}\right|$. Now if every man in $\mathcal{X}$ has a woman in $\mathcal{Y}^{\prime}$ as a partner in $\mu$, then every man in $\mathcal{X}^{\prime}$ must have a woman in $\mathcal{Y}$ in $\mu$. This gives us the lemma.

Lemma 6 Let $\mu$ and $\mu^{\prime}$ be two strong stable matchings in $\Omega_{N_{\mathcal{D}, \mathcal{M}}}$. If all men are given the better partners in the two matching $\mu$ and $\mu^{\prime}$, then the resultant matching, denoted as $\mu \wedge \mu^{\prime}$, is also a strong stable matching in $\Omega_{N_{\mathcal{D}, \mathcal{M}}}$.

Proof We first need to argue that $\mu \wedge \mu^{\prime}$ is really a matching. Suppose, for a contradiction, that both $m$ and $m^{\prime}$ are matched to $w$ in $\mu \wedge \mu^{\prime}$. Without loss of generality, let $m$ and $m^{\prime}$ be matched to $w$ in $\mu$ and $\mu^{\prime}$, respectively. By Lemma 5, since $m$ prefers matching $\mu$, then $w$ must prefer $\mu^{\prime}$. This, combined with the fact that $m^{\prime}$ also prefers $w$ to his partner in $\mu$, implies that $\left(m^{\prime}, w, d^{\prime}\right)$, where $\operatorname{dog} d^{\prime}$ always has $m^{\prime}$ as a partner in $\Omega_{N_{\mathcal{D}, \mathcal{M}}}$ is a blocking triple of degree 2 in $\mu$, a contradiction.

We now argue the stability of $\mu \wedge \mu^{\prime}$. Suppose that $(m, w, d)$ is a blocking triple of degree 3. Without loss of generality, let $m^{\prime}$ be the man who gets $w$ as a partner in $\mu$ and he prefers (or is indifferent to) $\mu$. In $\mu, w$ also strictly prefers $d$ to her assigned dog partner $d^{\prime}$, who always has $m^{\prime}$ as a partner in $\Omega_{N_{\mathcal{D}, \mathcal{M}}}$, in $\mu$. It is easy to see that man $m$ and $\operatorname{dog} d$ prefers $w$ and $m$, respectively, to their assigned partner in both $\mu$ and $\mu^{\prime}$. Therefore, $(m, w, d)$ is a blocking triple of degree 3 in $\mu$, a contradiction.

Finally, suppose $(m, w, d)$ is a blocking triple of degree 2 to $\mu \wedge \mu^{\prime}$. There are three cases to consider and their arguments are similar. We consider only one case. 
Suppose $m$ is the player who is indifferent. Let $\mu$ be the matching in which $m$ is matched to $w$ and $m$ prefers (or is indifferent to) $\mu$. Then $(m, w, d)$ is also a blocking triple of degree 2 in $\mu$, a contradiction.

The lemma below follows analogous arguments to those in the preceding one.

Lemma 7 Let $\mu$ and $\mu^{\prime}$ be two strong stable matchings in $\Omega_{N_{\mathcal{D}, \mathcal{M}}}$. If all women are given the better partners in the two matching $\mu$ and $\mu^{\prime}$, then the resultant matching, denoted as $\mu \vee \mu^{\prime}$, is a strong stable matching in $\Omega_{N_{\mathcal{D}, \mathcal{M}}}$.

Now, armed with Lemmas 6 and 7, we can introduce the lemma that establishes the distributive law of the lattice.

Lemma 8 Let $\mu, \mu^{\prime}$ and $\mu^{\prime \prime}$ be three strong stable matchings in $\Omega_{N_{\mathcal{D}}, \mathcal{M}}$. Then $\mu \wedge$ $\left(\mu^{\prime} \vee \mu^{\prime \prime}\right)=\left(\mu \wedge \mu^{\prime}\right) \vee\left(\mu \wedge \mu^{\prime \prime}\right)$ and $\mu \vee\left(\mu^{\prime} \wedge \mu^{\prime \prime}\right)=\left(\mu \vee \mu^{\prime}\right) \wedge\left(\mu \vee \mu^{\prime \prime}\right)$.

Proof Lemmas 6 and 7 establish that meet and join operations result in a strong stable matching in $\Omega_{N_{\mathcal{D}, \mathcal{M}}}$. The distributive law can be easily verified.

The correctness of Theorem 4 follows from Lemmas 6, 7 and 8.

\section{4 \#P-completeness of Strong Stable Matchings}

In this section, we present a reduction from the 2-party STABLE MARRIAGE problem to the 3-WAY KIDNEY TRANSPLANT problem. Counting the number of stable matchings in a stable marriage instance is \#P-complete, a fact established by Irving and Leather [13].

To build up some intuition, we first show how to "embed" a STABLE MARRIAGE instance $\Upsilon=(\mathcal{M}, \mathcal{W}, \mathcal{P})$ into a CIRCULAR STABLE MATCHING instance $\Upsilon^{\prime}=\left(\mathcal{M}^{\prime}, \mathcal{W}^{\prime}, \mathcal{D}^{\prime}, \mathcal{P}^{\prime}\right)$. For each player $p \in \mathcal{M} \cup \mathcal{W}$, we create a player $p^{\prime}$ and add her/him/it into the derived instance $\Upsilon^{\prime}$. Suppose a man $m_{i}^{\prime} \in \mathcal{M}^{\prime}$ is created based on $m_{i} \in \mathcal{M}$. We let him have the same preference as $m_{i}$. Precisely, supposing that $P\left(m_{i}\right)=w_{i 1} \succ w_{i 2} \succ \cdots \succ w_{i n}$, let $P^{\prime}\left(m_{i}^{\prime}\right)=w_{i 1}^{\prime} \succ w_{i 2}^{\prime} \succ \cdots \succ w_{i n}^{\prime}$. Furthermore, for each man $m_{i}^{\prime} \in M^{\prime}$, we create a $\operatorname{dog} d_{i}^{\prime}$ and add it into $\mathcal{D}^{\prime}$ with preference $P^{\prime}\left(d_{i}^{\prime}\right)=m_{i}^{\prime} \succ \cdots$. For a woman $w_{i}^{\prime} \in \mathcal{W}^{\prime}$, her preference is now for dogs, moreover, in her new preference, the indices are kept the same. To be precise, if $P\left(w_{i}\right)=m_{i 1} \succ m_{i 2} \succ \cdots \succ m_{i n}$, we make $P\left(w_{i}^{\prime}\right)=d_{i 1}^{\prime} \succ d_{i 2}^{\prime} \succ \cdots \succ d_{i n}^{\prime}$.

By this construction, it is easy to observe that the matching $\mu=\left\{\left(m_{j 1}, w_{j 1}\right)\right.$, $\left.\left(m_{j 2}, w_{j 2}\right), \ldots,\left(m_{j n}, w_{j n}\right)\right\}$ is stable in $\Upsilon$ if and only if the matching $\mu^{\prime}=$ $\left\{\left(m_{j 1}^{\prime}, w_{j 1}^{\prime}, d_{j 1}^{\prime}\right),\left(m_{j 2}^{\prime}, w_{j 2}^{\prime}, d_{j 2}^{\prime}\right), \ldots,\left(m_{j n}^{\prime}, w_{j n}^{\prime}, d_{j n}^{\prime}\right)\right\}$ is strongly stable in $\Upsilon^{\prime}$. A blocking pair $\left(m_{j k}, w_{j l}\right)$ in the former implies a blocking triple $\left(m_{j k}^{\prime}, w_{j l}^{\prime}, d_{j k}^{\prime}\right)$ of degree 2 in the latter. Conversely, there cannot be a blocking triple of degree 3 in $\mu^{\prime}$ (since every dog is matched to its top-ranked man). A blocking triple $\left(m_{j k}^{\prime}, w_{j l}^{\prime}, d_{j k}^{\prime}\right)$ of degree 2 implies that $\left(m_{j k}, w_{j l}\right)$ blocks $\mu$ as well. 
From the fact that the number of stable matchings in STABLE MARRIAGE can be exponential (see Knuth's book [15]), the fact that weak stable matchings are a superset of strong stable matchings, and the reduction given in Sect. 2.2, we establish:

Theorem 5 The number of weak and strong stable matchings in circular stable matching and 3-way kidney transplant problems can be exponential.

Unfortunately, the above construction of $\Upsilon^{\prime}$ is not a reduction, instead, it is merely an embedding. There is no guarantee that some other strong stable matching (in which dogs are not always matched to their top-ranked men) will not arise in $\Upsilon^{\prime}$. To prove the \#P-completeness, we need one more twist.

We transform $\Upsilon^{\prime}$ into a 3-WAY KIDNEY TRANSPLANT INSTANCE $\Upsilon^{\prime \prime}=\left(\mathcal{K}^{\prime \prime}, \mathcal{L}^{\prime \prime}\right)$ as follows. We first make a copy of every player in $\mathcal{M}^{\prime} \cup \mathcal{W}^{\prime} \cup \mathcal{D}^{\prime}$ and add it into $\mathcal{K}^{\prime \prime}$. For each $\operatorname{dog} d_{i}^{\prime \prime} \in \mathcal{K}^{\prime \prime}$, we create a set of guard players to restrict its possible successors in a strong stable matching. The idea here is similar to the one we used in the reduction of Sect. 2.3. We need an instance $\Upsilon_{d_{i}^{\prime \prime}}=\left(\mathcal{K}_{d_{i}^{\prime \prime}}, \mathcal{L}_{d_{i}^{\prime \prime}}\right)$ which has the properties: (1) it has four players, $k_{d_{i}^{\prime \prime}, j}^{\#}, 1 \leq j \leq 4$, and (2) it does not allow strong stable matching itself (see Appendix A for such an instance).

We embed $\Upsilon_{d_{i}^{\prime \prime}}$ into $\Upsilon^{\prime \prime}$ by altering the preferences of $d_{i}^{\prime \prime}$ and its associated three guard players as follows.

- $P^{\prime \prime}\left(d_{i}^{\prime \prime}\right)=m_{i}^{\prime \prime} \succ P_{\Upsilon_{d_{i}^{\prime \prime}}}\left(k_{d_{i}^{\prime \prime}, 1}^{\#}\right) \succ \cdots$, where $P_{\Upsilon_{d_{i}^{\prime \prime}}}\left(k_{d_{i}^{\prime \prime}, 1}^{\#}\right)$ is the preference list of $k_{d_{i}^{\prime \prime}, 1}^{\#}$ in the instance $\Upsilon_{d_{i}^{\prime \prime}}$.

- $P^{\prime \prime}\left(k_{d_{i}^{\prime \prime}, j}^{\#}\right)=P_{\Upsilon_{d_{i}^{\prime \prime}}}\left(k_{d_{i}^{\prime \prime}, j}^{\#}\right) \succ \cdots$, where $2 \leq j \leq 4$ and $P_{\Upsilon_{d_{i}^{\prime \prime}}}\left(k_{d_{i}^{\prime \prime}, j}^{\#}\right)$ is the preference list of $k_{d_{i}^{\prime \prime}, j}^{\#}$ in the instance $\Upsilon_{d_{i}^{\prime \prime}}$.

The intent here is to try to remove one player, $d_{i}^{\prime \prime}$ (who plays the role of $k_{d_{i}^{\prime \prime}, 1}^{\#}$ ) from $\Upsilon_{d_{i}^{\prime \prime}}$ to prevent a potential blocking triple in $\Upsilon_{d_{i}^{\prime \prime}}$ from blocking a strong stable matching in $\Upsilon^{\prime \prime}$.

After adding the $3 n$ guard players into $\mathcal{K}^{\prime \prime}$, we also have to update the preferences of the men and women who are the copies of those in $\mathcal{M}^{\prime} \cup \mathcal{W}^{\prime}$. Such a player, say, $m_{i}^{\prime \prime}$, replaces each woman $w_{j}^{\prime} \in \mathcal{W}^{\prime}$ with $w_{j}^{\prime \prime}$ in his list and attaches other players to the end of his list.

It can be checked that in all strong stable matchings in $\Upsilon^{\prime \prime}$, dogs have their topranked men as successors. Moreover, a matching $\mu=\left\{\left(m_{j 1}, w_{j 1}\right),\left(m_{j 2}, w_{j 2}\right), \ldots\right.$, $\left.\left(m_{j n}, w_{j n}\right)\right\}$ is stable in $\Upsilon$ if and only if a matching $\mu^{\prime \prime}=\left\{\left(m_{j 1}^{\prime \prime}, w_{j 1}^{\prime \prime}, d_{j 1}^{\prime \prime}\right)\right.$, $\left.\left(m_{j 2}^{\prime \prime}, w_{j 2}^{\prime \prime}, d_{j 2}^{\prime \prime}\right), \ldots,\left(m_{j n}^{\prime \prime}, w_{j n}^{\prime \prime}, d_{j n}^{\prime \prime}\right)\right\}$ is strongly stable in $\Upsilon^{\prime \prime}$. Therefore, the reduction from $\Upsilon$ to $\Upsilon^{\prime \prime}$ is correct. Using a similar and slightly more complicated gadget (of guard players), it is also possible to have a reduction from $\Upsilon$ to an instance of CIRCULAR STABLE MATCHING. We omit it here.

We conclude this section with the following theorem.

Theorem 6 It is \#P-complete to count the number of strong stable matchings in both circular stable matching and 3-way kidney transplant problems. 


\section{Conclusion}

We have left a complexity issue unanswered: existence of a weak stable circular matching. We were unable to come up with a NP-complete reduction, for there is no similar gadget (a small instance allowing no weak stable matchings) to the one we used in Sect. 2.3. Indeed, the reason may go deeper. Empirical evidence indicates that the number of weak stable circular matchings grows extraordinarily fast with the problem size. Eriksson, Sjöstrand and Strimling [5] conjectured that weak stable matchings always exist. This is why we remarked previously that finding one is probably not NP-complete.

Interestingly, Biró and McDermid [2] designed a small instance without weak stable matchings-under the assumption that players can truncate their preference lists. They were thus able to prove that the existence of weak stable matchings is also NP-complete in this context.

The obvious open questions are: when preferences are required to be complete, is there an instance in which no weak stable matchings exist? And if there is no such instance, is there a technique to prove their perennial existence.

Acknowledgement I thank Peter Winkler for many helpful discussions.

Open Access This article is distributed under the terms of the Creative Commons Attribution Noncommercial License which permits any noncommercial use, distribution, and reproduction in any medium, provided the original author(s) and source are credited.

\section{Appendix A: Examples of Small 3-way Kidney Transplant Instances Without Stable Matchings}

We mentioned in Sects. 2.3 and 5 that in the reductions, we need 3-WAY KIDNEY TRANSPLANT instances without weak/strong stable matchings. Two such instances are given in Tables 3 and 4 respectively.

Table 3 A 3-way kidney transplant instance of size 7 without weak stable matchings

Table 4 A 3-way kidney transplant instance of size 4 without strong stable matchings

\begin{tabular}{ll}
\hline Players & Preferences lists \\
\hline$k_{1}^{\#}$ & $k_{2}^{\#} \succ k_{5}^{\#} \succ k_{4}^{\#} \succ k_{6}^{\#} \succ k_{3}^{\#} \succ k_{7}^{\#}$ \\
$k_{2}^{\#}$ & $k_{4}^{\#} \succ k_{1}^{\#} \succ k_{6}^{\#} \succ k_{3}^{\#} \succ k_{5}^{\#} \succ k_{7}^{\#}$ \\
$k_{3}^{\#}$ & $k_{7}^{\#} \succ k_{2}^{\#} \succ k_{6}^{\#} \succ k_{1}^{\#} \succ k_{4}^{\#} \succ k_{5}^{\#}$ \\
$k_{4}^{\#}$ & $k_{5}^{\#} \succ k_{6}^{\#} \succ k_{3}^{\#} \succ k_{7}^{\#} \succ k_{1}^{\#} \succ k_{2}^{\#}$ \\
$k_{5}^{\#}$ & $k_{6}^{\#} \succ k_{7}^{\#} \succ k_{4}^{\#} \succ k_{1}^{\#} \succ k_{3}^{\#} \succ k_{2}^{\#}$ \\
$k_{6}^{\#}$ & $k_{3}^{\#} \succ k_{2}^{\#} \succ k_{1}^{\#} \succ k_{4}^{\#} \succ k_{5}^{\#} \succ k_{7}^{\#}$ \\
$k_{7}^{\#}$ & $k_{2}^{\#} \succ k_{4}^{\#} \succ k_{5}^{\#} \succ k_{3}^{\#} \succ k_{6}^{\#} \succ k_{1}^{\#}$ \\
\hline &
\end{tabular}

\section{Players} Preferences lists

\begin{tabular}{l}
$k_{1}^{\#}$ \\
$k_{2}^{\#}$ \\
$k_{3}^{\#}$ \\
$k_{4}^{\#}$ \\
\hline
\end{tabular}

$$
\begin{aligned}
& k_{3}^{\#} \succ k_{2}^{\#} \succ k_{4}^{\#} \\
& k_{4}^{\#} \succ k_{1}^{\#} \succ k_{3}^{\#} \\
& k_{4}^{\#} \succ k_{1}^{\#} \succ k_{2}^{\#} \\
& k_{3}^{\#} \succ k_{2}^{\#} \succ k_{1}^{\#}
\end{aligned}
$$




\section{References}

1. Abraham, D., Blum, A., Sandholm, T.: Clearing algorithms for barter exchange markets: Enabling nationwide kidney exchanges. In: ACM E-commerce (2007)

2. Biró, P., McDermid, E.: Three-sided stable matchings with cyclic preferences. Algorithmica (2009). doi:10.1007/s00453-009-9315-2, this issue

3. Boros, E., Gurvich, V., Jaslar, S., Krasner, D.: Stable matchings in three-sided systems with cyclic preferences. Discrete Math. 289(1-3), 1-10 (2004)

4. Cechlárová, K., Fleiner, T., Manlove, D.: The kidney exchange game. In: The 8th International Symposium on Operations Research in Slovenia, pp. 77-83 (2005)

5. Eriksson, K., Sjöstrand, J., Strimling, P.: Three-dimensional stable matching with cyclic preferences. Math. Soc. Sci. 52(1), 77-87 (2006)

6. Gale, D., Shapley, L.: College admissions and the stability of marriage. Am. Math. Monthly 69(1), 9-15 (1962)

7. Garey, M., Johnson, D.: Computers and Intractability. Freeman, New York (1979)

8. Gentry, S., Segev, D., Montgomery, R.: A comparison of populations served by kidney paired donation and list paired donation. Am. J. Transplant. 5(8), 1914-1921 (2004)

9. Gusfield, D., Irving, R.: The Stable Marriage Problem. MIT Press, Cambridge (1989)

10. Huang, C.-C.: Two's company, three's a crowd: stable family and threesome roommates problems. In: 15th Annual European Symposium on Algorithms (ESA) (2007)

11. Irving, R.: Stable marriage and indifference. Discrete Appl. Math. 48, 261-272 (1994)

12. Irving, R.: The cycle roommates problem: a hard case of kidney exchange. Inform. Process. Lett. 103, $1-4$ (2007)

13. Irving, R., Leather, P.: The complexity of counting stable marriages. SIAM J. Comput. 15, 655-667 (1986)

14. Karp, R.: Reducibility among combinatorial problems. In: Complexity of Computer Computations, pp. 85-103 (1972)

15. Knuth, D.: Mariages stables et leurs relations avec d'autre problèmes combinatoires. Les Presses de l'université de Montréal (1976)

16. Ng, C., Hirschberg, D.: Three-dimensional stable matching problems. SIAM J. Discrete Math. 4(2), 245-252 (1991)

17. Roth, A., Sonmez, T., Utku Unver, M.: Kidney exchange. Quart. J. Econ. 119(2), 457-488 (2004)

18. Roth, A., Sonmez, T., Utku Unver, M.: Increasing the opportunity of live kidney donation by matching for two and three way exchanges. Transplantation 81(5), 773-782 (2006)

19. Roth, A., Sonmez, T., Utku Unver, M.: Efficient kidney exchange: coincidence of wants in markets with compatibility-based preferences. Am. Econ. Rev. 97(3), 828-851 (2007)

20. Segev, D., Gentry, S., Waren, D., Reeb, B., Montgomery, R.: Kidney paired donation and optimizing the use of live donor organs. J. Am. Med. Assoc. 293(15), 1883-1890 (2005)

21. Subramanian, A.: A new approach to stable matching problems. SIAM J. Comput. 23(4), 671-700 (1994) 